Revue d'histoire de l'Amérique française

REVUE D.HISTOIRE DE L'AMÉRIQUE FRANÇAISE

\title{
Les parlementaires québécois depuis cinquante ans : continuité et renouvellement
}

\section{Réjean Pelletier}

Volume 44, numéro 3, hiver 1991

URI : https://id.erudit.org/iderudit/304898ar

DOI : https://doi.org/10.7202/304898ar

Aller au sommaire du numéro

\section{Éditeur(s)}

Institut d'histoire de l'Amérique française

\section{ISSN}

0035-2357 (imprimé)

1492-1383 (numérique)

Découvrir la revue

\section{Citer cet article}

Pelletier, R. (1991). Les parlementaires québécois depuis cinquante ans : continuité et renouvellement. Revue d'histoire de l'Amérique française, 44(3), 339-361. https://doi.org/10.7202/304898ar
Résumé de l'article

Empruntant à la fois aux théories élitistes de Mosca, Pareto et Michels, et aux théories pluralistes incarnées surtout par Robert Dahl, cet article met en lumière trois propositions principales. Il souligne d'abord la non-représentativité des représentants politiques, qui jouissent dans l'ensemble d'un statut social élevé, et, en second lieu, la non-homogénéité de ces représentants, selon leur appartenance à des partis politiques différents. Il met enfin en évidence le phénomène de la circulation des élites traduite en termes de renouvellement des parlementaires. Ces caractéristiques, que l'on retrouve également dans d'autres pays, sont confirmées par l'analyse des parlementaires québécois siégeant à l'Assemblée nationale depuis 1944. On peut ainsi conclure que, si la compétition politique engendre la sélection sociale, elle ne produit pas un personnel complètement homogène. $\mathrm{Au}$ contraire, des traits distinctifs caractérisent chacun des partis. En outre, depuis vingt ans, le renouvellement des parlementaires québécois est très marqué, ce qui contraste avec les périodes antérieures de stabilité.
Tous droits réservés @ Institut d'histoire de l'Amérique française, 1991
Ce document est protégé par la loi sur le droit d'auteur. L'utilisation des services d'Érudit (y compris la reproduction) est assujettie à sa politique d'utilisation que vous pouvez consulter en ligne.

https://apropos.erudit.org/fr/usagers/politique-dutilisation/ 


\title{
LES PARLEMENTAIRES QUÉBÉCOIS DEPUIS CINQUANTE ANS: CONTINUITÉ ET RENOUVELLEMENT ${ }^{1}$
}

\author{
RÉJEAN PELLETIER \\ Département de science politique \\ Université Laval
}

\section{RÉSUMÉ}

Empruntant à la fois aux théories élitistes de Mosca, Pareto et Michels, et aux théories pluralistes incarnées surtout par Robert Dahl, cet article met en lumière trois propositions principales. Il souligne d'abord la non-représentativité des représentants politiques, qui jouissent dans l'ensemble d'un statut social élevé, et, en second lieu, la non-homogénéité de ces représentants, selon leur appartenance à des partis politiques différents. Il met enfin en évidence le phénomène de la circulation des élites traduite en termes de renouvellement des parlementaires. Ces caractéristiques, que l'on retrouve également dans d'autres pays, sont confirmées par l'analyse des parlementaires québécois siégeant à l'Assemblée nationale depuis 1944. On peut ainsi conclure que, si la compétition politique engendre la sélection sociale, elle ne produit pas un personnel complètement homogène. Au contraire, des traits distinctifs caractérisent chacun des partis. En outre, depuis vingt ans, le renouvellement des parlementaires québécois est très marqué, ce qui contraste avec les périodes antérieures de stabilité.

\section{ABSTRACT}

Combining the elitist theories of Mosca, Pareto and Michels and the pluralist theories represented mostly by Robert Dahl, this article points to three main propositions. First, it underlines the non-representativity of political representatives, who generally enjoy a superior social status, and, secondly, their non-homogeneity, depending on political parties affiliation. Thirdly, it illustrates the phenomenon of elite circulation through the renewal of Legislatures. These characteristics, also present in other countries, are confirmed by the study of members of the Quebec National Assembly since 1944. One can thus conclude that if the political competition generates a social selection it does not create a totally homogeneous group, for members in each political party exhibit specific traits. The article also shows that, in the last twenty years, the rate of replacement of the Quebec Legislature has been important, in comparison with previous, more stable periods.

1 Communication présentée au Congrès annuel de l'Institut d'histoire de l'Amérique française, Saint-Jean-sur-Richelieu, 26-27 octobre 1990. Cette recherche a bénéficié du soutien financier du Conseil de recherches en sciences humaines du Canada et du Fonds FCAR du Québec. L'auteur tient à remercier Sylvain Ayotte, Luc Carrier, Bruno Lapointe, Chantal Nadeau et Manon Tremblay pour leur participation à ce projet. Le générique masculin utilisé ici n'a pour but que d'alléger le texte, tout en reconnaissant que les députées ont été trop peu nombreuses durant ce demi-siècle de vie politique québécoise.

RHAF, vol. 44, nº 3, hiver 1991 
Durant plusieurs siècles, on a cru que le pouvoir était distribué inégalement dans les sociétés. Outre le fait que les dirigeants étaient nettement moins nombreux que les dirigés, la source même du pouvoir ne pouvait favoriser qu'une minorité. Que celui-ci soit d'origine divine ou héréditaire, seules quelques personnes pouvaient en être investies.

La révolution démocratique en Europe devait remettre en cause ce paradigme. Elle postulait que tous les citoyens puissent partager ce pouvoir également, que celui-ci était ouvert à tous et que sa source reposait désormais sur la base, sur l'ensemble des citoyens, et non sur une autorité de droit divin ou un principe héréditaire. Cet optimisme démocratique était tempéré seulement par l'idée de représentation puisque tous ne pouvaient diriger en même temps la société: les citoyens devaient se choisir des représentants appelés à exercer le pouvoir pour une période déterminée.

\section{ÉLITISME ET PLURALISME}

À son tour, cet idéal démocratique devait être remis en cause par le courant élitiste classique incarné surtout par Gaetano Mosca, Vilfredo Pareto et Robert Michels. En dépit d'une façade démocratique qui s'était imposée progressivement au cours du XIX ${ }^{\mathrm{e}}$ siècle, le pouvoir, au tournant du siècle, n'appartenait toujours qu'à un groupe restreint. Bien plus, mêlant des considérations normatives à des situations de fait, les adeptes de ce courant de pensée estimaient que non seulement il en était ainsi, mais qu'il ne pouvait et ne devait en être autrement.

Robert D. Putnam a fort bien résumé les grands principes qui guident cette école de pensée ${ }^{2}$.

1) Le pouvoir politique, comme les autres biens sociaux, est distribué inégalement. Ainsi, on peut classer les individus selon la possession de certains biens comme la richesse, l'expertise, certaines qualités particulières, etc.

2) Essentiellement, les citoyens peuvent être divisés en deux groupes: ceux qui ont un pouvoir politique «significatif» et ceux qui n'en ont pas. La distribution du pouvoir peut donc être conçue en termes dichotomiques selon un partage entre gouvernants et gouvernés.

3) L'élite au pouvoir est homogène, unifiée et consciente d'ellemême: elle n'est donc pas une simple collection d'individus

2 Robert D. Putnam, The Comparative Study of Political Elites (Englewood Cliffs, Prentice-Hall, Contemporary Comparative Politics Series, 1976), 3-4. 
isolés. C'est plutôt une sorte de club exclusif: les gens se connaissent, partagent les mêmes valeurs, les mêmes intérêts et les mêmes attributs socio-économiques. James Meisel a résumé cette proposition dans sa théorie des trois $C$ : «group consciousness, coherence, and conspiracy», c'est-à-dire conscience de groupe, cohérence du groupe et partage d'intentions communes ${ }^{3}$.

4) L'élite se perpétue elle-même largement et est tirée d'un segment très exclusif de la société.

5) Pour toutes ces raisons, l'élite est essentiellement autonome, ne répondant qu'à elle-même des décisions qu'elle prend selon ses intentions et ses intérêts.

Selon la théorie élitiste, l'élection est un mécanisme qui permet à une collectivité de se donner des chefs légitimes appelés à prendre des décisions selon leur bon jugement et au nom de l'ensemble. Elle ne cherche pas à fournir l'image la plus fidèle possible du corps électoral, ni à charger les élus du mandat d'exécuter la volonté des électeurs. C'est plutôt la volonté des élus eux-mêmes qui a remplacé celle des citoyens.

Ces théories élitistes devaient à leur tour être remises en cause par le courant pluraliste bien incarné par Robert Dahl aux États-Unis ${ }^{4}$. Selon cette perspective, comme l'écrit Pierre Birnbaum, «les élites sont perçues comme des groupes dirigeants qui n'exercent leur pouvoir que dans un champ spécifique en n'ayant aucune influence sur les autres élites», ajoutant aussi que «les tenants du pluralisme se sont efforcés de démontrer, à l'aide de diverses méthodes, dont la méthode décisionnelle, l'autonomie sinon l'indépendance de chacun des champs» ${ }^{5}$.

Le pluralisme implique également une multiplicité de groupes défendant leurs intérêts dans la société et une multiplicité de points d'accès aux mécanismes de décision. C'est ainsi que chaque groupe, selon le contexte politique et sa force relative, peut parvenir au sommet de la hiérarchie. La conception pluraliste, selon François d'Arcy et Guy Saez, «met l'accent sur la circulation des flux de pouvoir et sur l'agencement complexe des groupes sociaux, les affiliations multiples des individus». Ils ajoutent: «La société s'organise d'elle-même en se

3 James Meisel, The Myth of the Ruling Class: Gaetano Mosca and the "Elite» (Ann Arbor, University of Michigan Press, 1962), 4.

4 Robert A. Dahl, Who Governs? Democracy and Power in an American City (New Haven, Yale University Press, 1961); également, Polyarchy. Participation and Opposition (New Haven, Yale University Press, 1970).

5 Pierre Birnbaum, Dimensions du pouvoir (Paris, Presses universitaires de France, coll. «Sociologie d'aujourd'hui», 1984), 209. 
différenciant selon une sorte de séparation sociale des pouvoirs qui fait des systèmes modernes des "polyarchies".» ${ }^{6}$ Une telle conception s'oppose à celle de $\mathrm{C}$. Wright Mills à l'effet qu'il existe une structure du pouvoir aux États-Unis constituée de l'alliance entre l'élite économique, politique et militaire ${ }^{7}$.

En somme, en reprenant l'excellente distinction d'Eva EtzioniHalevy, on peut dire que les théories pluralistes ont amplifié la dispersion du pouvoir et la multiplicité des élites en compétition dans les démocraties, alors que certains théoriciens élitistes ont plutôt amplifié la nature concertée ou consensuelle du pouvoir, même en démocratie ${ }^{8}$.

Le texte qui suit ne cherche pas à trancher entre ces deux grands courants. Tout en me rattachant aux théories élitistes, je voudrais également mettre en lumière certains éléments du courant pluraliste. Puisant à l'une ou à l'autre source, je tenterai de vérifier les trois propositions suivantes:

1) En conformité avec les théories élitistes, je voudrais souligner tout d'abord la non-représentativité des représentants. Ce concept de non-représentativité appelle un certain nombre de précisions. Comme le faisait déjà remarquer $\mathrm{T}$. $\mathrm{B}$. Bottomore, la minorité au pouvoir, de par sa nature même, ne peut être représentative en tous points de la majorité, au moins par le fait qu'elle détient un pouvoir plus grand que ceux qu'elle représente ${ }^{9}$. Plus précisément, si l'on reprend les distinctions déjà établies par Hanna Pitkin, le concept de représentation peut s'entendre en termes d'activité dans le sens où un parlementaire agit au nom d'un groupe: ses actions ou ses opinions doivent alors être en accord avec les désirs, les besoins ou les intérêts de ceux pour lesquels il agit. La représentation peut également prendre une forme symbolique dans le sens où les représentants sont le reflet, en termes de caractéristiques sociales (sexe, origine ethnique, couleur, etc.), de ceux et celles qu'ils représentent. Une telle conception de la représentation implique que la seule présence des membres d'un groupe est suffisante pour représenter les intérêts de ce groupe. C'est un rapport de ressemblance ou d'équivalence qui est alors mis en évidence ${ }^{10}$.

6 François d'Arcy et Guy Saez, «De la représentation», François d'Arcy, dir., $L a$ représentation (Paris, Economica, 1985), 21.

7 C. Wright Mills, The Power Elite (New York, Oxford University Press, 1956).

8 Eva Etzioni-Halevy, Fragile Democracy. The Use and Abuse of Power in Western Societies (New Brunswick, Transaction Publishers, 1989), 17.

9 T. B. Bottomore, Élites et société (Paris, Stock, 1967), 129.

10 Hanna Pitkin, The Concept of Representation (Berkeley, University of California Press, 1967), 60-143. 
C'est plutôt cette seconde conception que je vais privilégier ici. Peut-être faudrait-il parler dans ce cas de non-similarité entre les élus et leurs commettants. Plus exactement, je m'arrêterai à certains attributs sociaux qui caractérisent cette élite politique afin de montrer que la compétition politique engendre la sélection sociale. Comme l'écrivait Daniel Gaxie à propos de la situation française:

La représentation parlementaire fournit ainsi une image inversée de la structure sociale puisque plus des trois quarts des députés sont issus du décile le plus favorisé - socialement et culturellement - de la population. Sous ce rapport, la compétition politique intéresse (dans tous les sens) avant tout les agents situés au sommet de la hiérarchie sociale ou, mieux, les différentes fractions des classes supérieures. Corrélativement, les caractéristiques sociales du personnel politique deviennent «plus aristocratiques» à mesure que l'on s'élève dans la hiérarchie de pouvoir et de prestige des postes en compétition. ${ }^{11}$

Et il concluait en ces termes: «Dans les conditions d'un marché politique symboliquement hiérarchisé, la concurrence politique ne peut être qu'une forme de sélection sociale.»

2) Contrairement à l'un des grands principes de la théorie élitiste et en conformité avec certains éléments du courant pluraliste, je voudrais également souligner la non-homogénéité de ces représentants selon leur appartenance à des partis politiques différents. Cayrol et Perrineau, analysant l'élite politique française, avaient déjà remarqué certaines «déviations» entre gaullistes et giscardiens par delà une grande homogénéité au sein de l'élite. Surtout, les données recueillies semblaient refléter des processus différents de mobilité sociale entre les socialistes d'une part et les gaullistes et giscardiens d'autre part ${ }^{12}$. D'autres, comme Daniel Gaxie, ont plutôt souligné la possession différenciée du capital culturel et du capital économique au sein de l'élite politique. S'appuyant sur Bourdieu $^{13}$ qui distingue, chez les classes moyennes et supérieures, un pôle intellectuel dominé, caractérisé par la prédominance relative du capital culturel par rapport au capital économique, et un pôle économique dominant où le capital économique l'emporte

11 Daniel Gaxie, «Les logiques du recrutement politique», Revue française de science politique, 30,1 (février 1980): 6.

12 Roland Cayrol et Pascal Perrineau, «Governing Elites in a Changing Industrial Society: the Case of France», Moshe M. Czudnowski, ed., Does Who Governs Matter? Elite Circulation in Contemporary Societies (De Kalb, Northern Illinois University Press, 1982), 90124.

13 Pierre Bourdieu et Monique de Saint-Martin, «Anatomie du goût», Actes de la recherche en sciences sociales, 5 (octobre 1976): 4-81. 
sur le capital culturel, Gaxie conclut que «la compétition politique exprime principalement l'antagonisme entre le pôle intellectuel et le pôle économique des classes dominantes» ${ }^{14}$. Ce que je voudrais vérifier dans ce texte, c'est plutôt la possession différenciée d'un capital culturel et d'un capital économique qui recoupe les différences inter-partisanes.

3) Les théoriciens élitistes classiques comme Mosca et Pareto ont accordé un rôle important aux processus de reproduction, de changement, de renouvellement et de désintégration des élites. Cette «circulation des élites» est considérée comme une variable majeure dans l'explication de la continuité et discontinuité, du fonctionnement et «malfonctionnement» des systèmes politiques ${ }^{15}$. Rejoignant d'une certaine façon cette théorie sur la circulation des élites, certains, comme Polsby ${ }^{16}$, Eliassen et Pedersen ${ }^{17}$, ont plutôt insisté sur l'institutionnalisation d'une assemblée représentative exprimée en termes de professionnalisation du personnel politique et de longévité de la carrière. À partir des travaux de Samuel Huntington qui avait cherché à préciser et enrichir ce concept d'institutionnalisation ${ }^{18}$, ces auteurs ont tenté d'opérationnaliser davantage les variables énoncées par Huntington. En relation avec ces concepts de circulation des élites, d'institutionnalisation d'une assemblée et de professionnalisation du personnel, je voudrais m'arrêter ici au degré d'ouverture de la classe politique traduite en termes de renouvellement des parlementaires, ainsi qu'à la recomposition subséquente des élites partisanes.

\section{LE CHAMP D'ANALYSE}

J'essaierai de vérifier ces trois propositions en les appliquant à l'élite politique québécoise identifiée ici à l'ensemble des parlementaires, mais sans distinction de postes occupés par l'un ou par l'autre. Cette définition exclut les leaders non élus des partis politiques ainsi que tous les hauts fonctionnaires. Elle exclut également les candidats qui n'ont pas remporté la victoire. Le critère premier est donc électoral: il faut avoir été élu au cours d'une élection générale.

14 Daniel Gaxie, op. cit., 36.

15 Voir Maurizio Cotta, «The Italian Political Class in the Twentieth Century: Continuities and Discontinuities», Moshe M. Czudnowski, ed., op. cit., 156-159.

16 Nelson Polsby, «The Institutionalization of the U.S. House of Representative», American Political Science Review, 62,1 (March 1968).

17 K. Eliassen et M. Pedersen, «Professionalization of Legislatures: Long-Term Change in Political Recruitment in Denmark and Norway», Comparative Studies in Society and History, 20,2 (April 1978): 286-318.

18 Samuel P. Huntington, Political Order in Changing Societies (New Haven and London, Yale University Press, 1968). 
Dans ce texte, j'emploie d'une façon indifférenciée les concepts d'élite politique, de classe politique, d'élite dirigeante, de personnel politique, tous identifiés à l'ensemble des députés élus à l'Assemblée nationale du Québec.

Ma stratégie de vérification repose sur une perspective diachronique: je retiens, en effet, sept moments principaux d'intervention, chacun étant caractérisé par un changement de gouvernement, à l'exception de l'élection de 1956. En mettant ainsi l'accent sur près d'un demi-siècle d'histoire politique, il sera possible de mieux dégager ce qui relève de la permanence et du changement au sein de l'élite politique québécoise. La première élection, celle de 1944, marque l'arrivée au pouvoir pour une seconde fois de l'Union nationale dirigée par Maurice Duplessis. L'élection de 1956, souvent décriée pour ses abus et ses fraudes, traduit une longue fidélité de l'électorat québécois au même parti unioniste. Par contre, l'arrivée au pouvoir des libéraux, sous le leadership de Jean Lesage, inaugure ce que l'on a appelé la Révolution tranquille. Dès 1966, l’UN revient au pouvoir, mais c'est un parti renouvelé, alors dirigé par Daniel Johnson, qui affronte l'électorat.

On a l'habitude de considérer l'élection de 1970 comme une élection du réalignement: retour des libéraux au pouvoir, dirigés pour la première fois par Robert Bourassa, déclin de l'UN et arrivée de deux nouveaux partis sur la scène parlementaire, le Parti québécois et le Ralliement créditiste. Dès 1976, le Parti québécois, sous le leadership de René Lévesque, est porté au pouvoir. Enfin, le Parti libéral, dirigé pour la seconde fois par Robert Bourassa, obtient à nouveau la victoire à l'élection de 1985.

Je m'arrêterai donc à ces sept élections afin de vérifier les trois propositions énoncées précédemment ${ }^{19}$.

\section{NON-REPRÉSENTATIVITÉ DES REPRÉSENTANTS}

Le caractère élitiste et non représentatif des parlementaires aussi bien au Québec que dans d'autres pays a maintes fois été démontré. Je m'y attarderai assez peu ici, sinon pour analyser certains attributs socio-économiques qui traduisent bien ce caractère non représentatif du personnel politique.

19 La plupart des données présentées ici ont été tirées du Répertoire des parlementaires québécois, 1867-1978 (Québec, Bibliothèque de la législature, Service de documentation politique, 1980) et de sa mise à jour. Cet ouvrage présente une biographie de tous les parlementaires québécois en rassemblant, à partir d'une grille uniforme, des informations sur leur carrière professionnelle, politique et parlementaire, ainsi que sur un certain nombre de caractéristiques socio-économiques. 
On peut s'arrêter à deux modèles opposés sur la composition des élites ${ }^{20}$. Chacun de ces modèles constitue un idéal-type qui ne peut correspondre parfaitement à la réalité. Le premier, appelé le modèle de l'indépendance, postule une relation négligeable entre le statut socioéconomique et le statut politique. Ainsi chaque citoyen peut accéder à l'élite politique, indépendamment de son occupation, de son éducation, de son âge, de son sexe, de sa religion, etc. Bien plus, chaque catégorie sociale serait adéquatement représentée au sein de l'élite. Le modèle opposé postule au contraire une corrélation parfaite entre la place de l'individu dans la stratification politique et sa place dans la hiérarchie sociale, si bien qu'une caste privilégiée sur le plan socio-économique monopolise le leadership politique. Ce modèle, dit de l'agglutination, suppose une fusion des valeurs, en ce sens que les plus puissants sont également les plus riches, les plus prestigieux et présumément les plus sages.

Si la réalité ne se conforme pas exactement à ces modèles, il est tout de même facile de montrer, à la suite de Mosca, que l'élite politique jouit d'un statut social élevé. Analysant les nouveaux élus socialistes français de 1981 et les comparant à l'ensemble des députés du PS, Annie Collovald conclut à un élitisme constant, tout en soulignant que le epremier principe de structuration des variables sociales se constitue sur la base du capital scolaire pour les nouveaux élus socialistes ${ }^{21}$. Qu'en est-il de l'élite politique québécoise?

De 1944 jusqu'en 1960, la Chambre est composée d'une faible majorité d'universitaires plutôt que de non-universitaires (voir le tableau 1). Ce qui peut sembler étonnant si l'on tient compte du fait que l'Union nationale domine largement l'Assemblée élue (près des trois quarts des députés, sauf en 1944 où elle n'obtient que 53\% des sièges) et que ce parti se démarque par sa majorité de députés qui n'a pas fréquenté l'université. C'est alors le Parti libéral qui, malgré sa faible représentation, réussit à donner une coloration universitaire à la Chambre.

Cette situation va persister même après l'élection de 1960 au moment où le Parti libéral réussit à déloger l'UN et à s'emparer du pouvoir. On constate alors que la présence des non-universitaires demeure encore forte à l'Assemblée et que, bien plus, cette présence s'accroît légèrement au sein du PLQ. En ce sens, 1960 ne marque pas une rupture par rapport aux années antérieures si l'on tient compte du seul critère de la scolarité.

20 Voir Robert D. Putnam, op. cit., 21-22.

21 Annie Collovald, «La République du militant. Recrutement et filières de la carrière politique des députés», Pierre Birnbaum, dir., Les élites socialistes au pouvoir, 1981-1985 (Paris, Presses universitaires de France, 1985), 37-52. 
TABLEAU 1

Niveau de scolarité des députés québécois, 1944-1985

\begin{tabular}{ccclccc}
\hline & \multicolumn{2}{c}{ Non-universitaire } & \multicolumn{2}{l}{ Universitaire* } & \multicolumn{2}{c}{ NSP** } \\
\hline & $\mathbf{N}$ & $\%$ & N & \% & N & $\%$ \\
\cline { 2 - 7 } 1944 & 42 & 46,2 & 47 & 51,6 & 2 & 2,2 \\
1956 & 45 & 48,4 & 47 & 50,5 & 1 & 1,1 \\
1960 & 41 & 43,2 & 54 & 56,8 & - & - \\
1966 & 29 & 26,9 & 79 & 73,1 & - & - \\
1970 & 27 & 25,0 & 80 & 74,1 & 1 & 0,9 \\
1976 & 22 & 20,0 & 88 & 80,0 & - & - \\
1985 & 30 & 24,6 & 92 & 75,4 & - & - \\
\hline
\end{tabular}

* Qui a fréquenté l'université, qu'il y ait obtenu ou non un diplôme. En outre, tous les détenteurs d'un baccalauréat ès arts (B.A.) ont été classés dans cette catégorie (diplôme décerné à l'époque par l'université).

** Il faudrait normalement ranger les députés de cette catégorie parmi les non-universitaires, puisque les renseignements fournis indiquent habituellement qu'ils ont étudié à l'école paroissiale, sans plus de précision.

Il faudra attendre l'élection de 1966 et le réalignement de 1970 pour que la formation universitaire, comme je l'ai déjà souligné ailleurs ${ }^{22}$, devienne pratiquement une condition d'entrée à l'Assemblée nationale. Si, à l'aube de la Révolution tranquille, $43 \%$ des députés n'ont pas reçu une formation universitaire, ce nombre est réduit à $25 \%$ en 1970 pour se stabiliser par la suite autour de 20-25\% (à l'exception de 1981 où il se situe à $16 \%$ seulement). En 1966, la présence d'universitaires est encore plus marquée qu'autrefois chez les libéraux, alors que les députés unionistes deviennent de plus en plus scolarisés, ce qui traduit un renouvellement important du personnel au sein de l'Union nationale. Les partis (aussi bien le PLQ que l'UN et le PQ) recrutent donc leurs candidats au sein d'une élite intellectuelle nullement représentative de l'ensemble de la population. Comme l'écrivait déjà Robert Boily, «la très grande majorité de nos hommes politiques a reçu une formation que seule une minorité pouvait recevoir au Québec»23.

Par delà ce caractère élitiste, il faut également souligner que ce nouveau critère d'entrée dans la classe politique est relié en même temps à l'accroissement de la scolarité parmi la population, même si un écart important subsiste toujours entre les députés et leurs

22 Réjean Pelletier, «Le personnel politique», Recherches sociographiques, 25,1 (janvier-avril 1984): 95 .

23 Robert Boily, «Les hommes politiques du Québec, 1867-1967», Vincent Lemieux, dir., Personnel et partis politiques au Québec (Montréal, Boréal Express, 1982), 100. 
commettants (de 7,5\% de la population québécoise - de 15 ans et plus ne fréquentant plus l'école - qui a reçu une formation universitaire en 1971 à 13,5\% en 1981). Surtout, un niveau de scolarité élevé devient l'une des avenues les plus importantes de la mobilité verticale dans la société moderne ${ }^{24}$. Comme voie de promotion sociale, le diplôme n'est pas seulement un instrument d'accès à une profession, il ouvre également des portes autrement difficiles à percer et en ferme d'autres à ceux qui ne jouissent pas de ce capital scolaire. Tel semble être le cas, à l'heure actuelle, de l'accès à l'élite politique non seulement au Québec, mais aussi dans d'autres pays ${ }^{25}$.

Ce critère d'entrée reflète également l'importance accordée à l'éducation dans les sociétés modernes. Selon les types de problèmes qui surgissent dans une société, selon la priorité qu'on leur accorde, selon les types de solutions proposées, non seulement pourra-t-on privilégier une élite dirigeante scolarisée, mais aussi valoriser davantage des diplômes supérieurs et plus diversifiés qu'autrefois. C'est ainsi qu'une formation en droit, longtemps privilégiée comme type de formation au sein du personnel politique, a perdu son importance première au profit des détenteurs de maîtrises et de doctorats dans d'autres disciplines. Ce phénomène, comme le rappelaient Prewitt et McAllister, semble traduire le passage d'une époque de simple régulation, où les avocats pouvaient utiliser davantage leur expertise juridique, à une époque plus préoccupée par la gestion de la fiscalité et de l'économie, par les problèmes sociaux et éducationnels, bref par des questions qui font davantage appel à de nouveaux experts en économie, en administration, en sciences sociales, détenteurs de diplômes élevés témoignant de cette expertise ${ }^{26}$. Cette nouvelle élite caractéristique de la société post-industrielle se retrouve aussi au Québec (voir le tableau 2).

En somme, le dernier demi-siècle y a été marqué par les traits suivants en matière de scolarité:

- disparition du primaire comme dernier niveau d'étude. Le faible contingent classé dans cette catégorie (en moyenne $8,4 \%$ de l'ensemble des députés sous le régime duplessiste) se maintient jusqu'en 1960, puis décroît rapidement et disparaît complètement par la suite;

24 Voir Werner Kaltefleiter, «The Recruitment Market of the German Political Elite», Heinz Eulau et Moshe M. Czudnowski, eds., Elite Recruitment in Democratic Polities. Comparative Studies Across Nations ([Beverly Hills], Sage Publications, 1976), 244.

25 Pour la France, consulter Brigitte Gaïti, «Politique d'abord: le chemin de la réussite ministérielle dans la France contemporaine», Pierre Birnbaum, dir., op. cit., 65; pour les ÉtatsUnis, voir Kenneth Prewitt et William McAllister, «Changes in the American Executive Elite, 1930-1970», Heinz Eulau et Moshe M. Czudnowski, eds., op. cit., 111-112.

26 K. Prewitt et W. McAllister, op. cit., 118. 
- déclin des études de niveau secondaire, surtout depuis les élections de 1966 et 1970 , avec une stabilisation de ce groupe autour de $18 \%$ depuis 1976 et une remontée à $24 \%$ en 1985 (comme en 1970);

- déclin de la formation en droit dont la représentation s'est stabilisée autour de $16-18 \%$ depuis 1970 , alors qu'elle se situait en moyenne à $29 \%$ de l'ensemble des députés durant la période duplessiste et atteignait encore 30\% en 1962;

- diversification des professions ${ }^{27}$, déjà amorcée sous le régime duplessiste avec l'arrivée d'agronomes, de comptables ou de pharmaciens, et qui s'accentue à partir de 1966. Désormais, des ingénieurs, des économistes, des professeurs, des journalistes se retrouvent en plus grand nombre qu'autrefois au sein de la Chambre;

- croissance importante des détenteurs de maîtrise et de doctorat, passant de $0 \%$ en 1956 à $13 \%$ en $1970,24,5 \%$ en 1976 et $30 \%$ en 1981 pour ensuite reculer à $22 \%$ en 1985 .

TABLEAU 2

Types de scolarité universitaire des députés québécois, 1944-1985

\begin{tabular}{rrrrrrrrr}
\hline & \multicolumn{2}{l}{ Droit } & \multicolumn{2}{c}{ Médecine } & \multicolumn{2}{c}{ Autre $\mathbf{1}^{\text {er }}$ cycle } & \multicolumn{2}{c}{$\begin{array}{c}\text { Maîtrise et } \\
\text { doctorat }\end{array}$} \\
\hline & N & \%* & N & \% & N & \% & N & \% \\
\hline 1944 & 30 & 33,0 & 7 & 7,7 & 9 & 9,9 & 1 & 1,1 \\
1956 & 22 & 23,7 & 10 & 10,8 & 15 & 16,1 & - & - \\
1960 & 25 & 26,3 & 8 & 8,4 & 19 & 20,0 & 2 & 2,1 \\
1966 & 27 & 25,0 & 8 & 7,4 & 36 & 33,3 & 8 & 7,4 \\
1970 & 20 & 18,5 & 6 & 5,6 & 40 & 37,0 & 14 & 13,0 \\
1976 & 16 & 14,5 & 3 & 2,7 & 42 & 38,2 & 27 & 24,5 \\
1985 & 20 & 16,4 & 2 & 1,6 & 43 & 35,2 & 27 & 22,1 \\
\hline
\end{tabular}

* Pourcentage calculé à partir de l'ensemble des députés élus à chaque élection.

La situation québécoise se conforme donc au modèle général du passage obligé par l'université pour accéder à l'élite politique. Mais la

27 Comme on pourra le constater, le terme «profession» n'est pas réservé dans ce texte aux seules professions libérales, et n'est pas toujours sanctionné par un diplôme universitaire, bien que ce soit le plus souvent le cas. 
scolarisation est aussi reliée au statut socio-économique. En dépit d'une volonté de démocratisation du contenu de l'enseignement et des voies d'accès à l'éducation, les travaux de Bourdieu et Passeron ${ }^{28}$ ont bien montré que l'école agit comme' un filtre et que l'accès à l'éducation dépend encore du statut social. Si l'on peut établir un lien évident entre niveau d'instruction et sélection politique, qu'en est-il du statut social défini en termes d'occupation? Le cas québécois se conforme-t-il au modèle général de sélection de l'élite dirigeante parmi les occupations de niveau élevé?

Analysant l'élite politique allemande, Kaltefleiter a remarqué qu'elle se recrute dans une variété de secteurs occupationnels, bien que certains secteurs soient privilégiés comme les affaires, la fonction publique et les milieux académiques ${ }^{29}$. Le Québec se distingue de ce modèle sur certains points. Ainsi la «République des fonctionnaires» ou la «République administrative», pour reprendre les expressions de Pierre Birnbaum, qui s'est installée progressivement en France depuis les mutations institutionnelles de 1958, n'a pas pris racine au Québec. Certes, un plus grand nombre de fonctionnaires qu'autrefois font le saut de l'administratif au politique - si tant est qu'une telle distinction soit toujours pertinente - mais ce passage est sans commune mesure avec la situation française ou allemande.

La filière québécoise est souvent différente (voir le tableau 3). Les milieux d'affaires et la haute administration sont sur-représentés. Mais il faut souligner que ce sont essentiellement des propriétaires de PME (industriels ou commerçants) qui composent cette catégorie. Avant 1960, l'Union nationale a accueilli un plus grand nombre de petits entrepreneurs que le Parti libéral: ils ont le plus souvent formé un noyau important de notables locaux, bien implantés dans leur milieu et ardents défenseurs du nationalisme traditionnel. En agissant comme véhicules de l'idéologie dominante, ils ont pu préserver leur position sociale et leur leadership local.

Par la suite, des modifications importantes vont se produire au sein du personnel politique, surtout à partir de 1966. Le déclin des petits entrepreneurs sera compensé par la montée des professionnels. Mais le droit ne constitue plus la norme générale et son emprise a diminué au profit d'une diversification des professions. Ce sont surtout les professionnels de la culture (professeurs, journalistes) qui vont s'imposer au cours des années 1970 et, plus particulièrement, avec

28 Pierre Bourdieu et Jean-Claude Passeron, Les héritiers. Les étudiants et la culture (Paris, Éditions de Minuit, 1985) (c1964).

29 Werner Kaltefleiter, op. cit., 250-254. 
TABLEAU 3

Occupation des députés québécois, 1944-1985*

\begin{tabular}{|c|c|c|c|c|c|c|c|c|c|c|c|c|}
\hline & \multicolumn{2}{|c|}{$\begin{array}{l}\text { Haute admistration. } \\
\text { Industriels, PME }\end{array}$} & \multicolumn{2}{|c|}{$\begin{array}{l}\text { Cadres adminis- } \\
\text { tratifs intermédiaires } \\
\text { et inférieurs }\end{array}$} & \multicolumn{2}{|c|}{ Droit } & \multicolumn{2}{|c|}{ Culture } & \multicolumn{2}{|c|}{$\begin{array}{c}\text { Autres } \\
\text { professions }\end{array}$} & \multicolumn{2}{|c|}{$\begin{array}{c}\text { Employés } \\
\text { bureau, ouvriers, } \\
\text { cultivateurs }\end{array}$} \\
\hline & $\mathbf{N}$ & $\%$ & $\mathbf{N}$ & $\%$ & $\mathbf{N}$ & $\%$ & $\mathbf{N}$ & $\%$ & $\mathbf{N}$ & $\%$ & $\mathbf{N}$ & $\%$ \\
\hline 1944 & 27 & 29,7 & 12 & 13,2 & 28 & 30,8 & 2 & 2,2 & 10 & 11,0 & 12 & 13,2 \\
\hline 1956 & 34 & 36,6 & 13 & 14,0 & 20 & 21,5 & 3 & 3,2 & 14 & 15,1 & 9 & 9,7 \\
\hline 1960 & 32 & 33,7 & 14 & 14,7 & 23 & 24,2 & 2 & 2,1 & 17 & 17,9 & 7 & 7,4 \\
\hline 1966 & 21 & 19,4 & 17 & 15,7 & 28 & 25,9 & 11 & 10,2 & 22 & 20,4 & 9 & 8,3 \\
\hline 1970 & 22 & 20,4 & 31 & 28,7 & 17 & 15,7 & 10 & 9,3 & 20 & 18,5 & 8 & 7,4 \\
\hline 1976 & 20 & 18,2 & 24 & 21,8 & 14 & 12,7 & 30 & 27,3 & 11 & 10,0 & 11 & 10,0 \\
\hline 1985 & 30 & 24,6 & 30 & 24,6 & 23 & 18,9 & 17 & 13,9 & 12 & 9,8 & 10 & 8,2 \\
\hline
\end{tabular}


l'arrivée du Parti québécois au pouvoir en 1976, à l'instar de ce qui a pu survenir en France avec la venue de la gauche au pouvoir en $1981^{30}$. Si l'on a pu parler du retour des intellectuels dans le cas français, il faudrait plutôt parler de l'arrivée pour la première fois des intellectuels au pouvoir (identifiés ici aux professionnels de la culture) dans le cas québécois.

Même si les professions sont de plus en plus diversifiées, on peut admettre une sorte d'équivalence entre elles en ce sens qu'on a fini par reconnaître les nouvelles professions. Leur commune appartenance à des matières intellectuelles, sanctionnées habituellement par des diplômes universitaires, a pu suffire comme critère d'acceptation. Ce sont alors davantage les études universitaires que l'appartenance à certaines professions qui constituent la barrière d'accès à l'élite politique. Dès que l'on a franchi cette barrière, on accède à un même niveau (selon les termes popularisés par Edmond Goblot ${ }^{31}$ ) où s'estompent ces différences de professions: le passage obligé par l'université constitue précisément cette barrière, pour ceux qui ne l'ont pas encore franchie, et ce niveau qui uniformise entre eux ceux qui l'ont franchie. Évidemment, études universitaires et exercice d'une profession vont habituellement de pair, mais le niveau universitaire constitue la nouvelle barrière d'accès à l'élite dirigeante par delà la diversité nouvelle des professions. Une telle conclusion nous rapproche davantage du modèle de l'agglutination que de celui de l'indépendance.

\section{NON-HOMOGÉNÉITÉ DES REPRÉSENTANTS}

Si la concurrence politique constitue une forme de sélection sociale qui se traduit par le caractère non représentatif de l'élite politique, elle n'entraîne pas nécessairement une parfaite homogénéité de cette élite. Certes, on peut y partager les mêmes valeurs, défendre les mêmes intérêts, mais on ne possède pas nécessairement les mêmes attributs socio-économiques. Ce que je voudrais mettre en évidence ici, c'est la possession différenciée d'un capital culturel et d'un capital économique au sein de la classe dirigeante en fonction des clivages partisans.

Selon certains, une forte homogénéité de l'élite va renforcer son intégration; à l'inverse, une trop grande hétérogénéité peut engendrer des problèmes politiques. Surtout, l'étude des attributs socio-économiques des membres de l'élite servirait davantage comme un

30 Comme le rappelait Brigite Gaïti, op. cit., 56.

31 Edmond Goblot, La barrière et le niveau (Paris, Presses universitaires de France, coll. SUP «Le sociologue», 1967) (c1925). 
indicateur de la structure du pouvoir social que comme un moyen de prédire le comportement de ces membres ${ }^{32}$. Ainsi, la montée des intellectuels ou des entrepreneurs au sein de l'élite politique serait la manifestation de l'importance de ces groupes dans la société à un moment donné. Si cette théorie s'avère fondée, on devrait pouvoir vérifier la montée des entrepreneurs et la présence plus grande des détenteurs d'un capital économique au sein de la classe politique québécoise à un moment où, comme c'est le cas depuis quelques années, l'entrepreneurship est valorisé, où les modèles proposés sont économiques, où l'on appelle au désengagement de l'État au profit du secteur privé. Il en serait de même à l'époque de Duplessis où le conservatisme social prôné par l'Union nationale s'accompagne d'une modernisation économique qui se fait progressivement, bien qu'elle soit davantage le fait de la grande entreprise que des petits entrepreneurs ${ }^{33}$.

Tout d'abord, peut-on vérifier la possession différenciée d'un capital culturel et d'un capital économique chez le personnel politique québécois depuis le réalignement de 1970? Peut-on opposer, en d'autres termes, l'intellectuel et l'entrepreneur? Si l'on s'attache aux champs d'activités professionnelles dans lesquels sont impliqués les parlementaires québécois, il est possible de déceler des différences importantes entre les députés libéraux et les députés péquistes, comme le montre le tableau 4. Chez les premiers, on valorise davantage les champs économique et administratif, bien que le champ social soit tout de même représenté par un député libéral sur quatre. Chez les seconds, on peut noter la présence fort importante du champ culturel qui l'emporte nettement sur tous les autres champs, même si à peu près un député péquiste sur cinq provient également du champ social. Ces différences entre les deux partis s'affirment depuis quinze ans, plus marquées en 1976, mais continuellement présentes depuis la création du Parti québécois: celui-ci a bénéficié, jusqu'en 1985, d'un appui important auprès des étudiants, des professeurs, des journalistes et des artistes, et il a toujours été identifié aux travailleurs du champ culturel et surtout au secteur de l'enseignement, face à un Parti libéral plus étroitement lié au monde des affaires et de l'administration ainsi qu'au champ économique.

À cet égard, le Parti québécois s'apparente aux socialistes français où domine le pôle intellectuel, alors que le Parti libéral s'apparente à la droite française où s'impose le pôle économique. Comme le faisait

32 Voir, à ce sujet, Robert D. Putnam, op. cit., 43.

33 Sur ce thème, voir Réjean Pelletier, Partis politiques et société québécoise. De Duplessis à Bourassa, 1944-1970 (Montréal, Québec/Amérique, 1989), 53-59. 
TABLEAU 4

Champs d'activités des députés, selon le parti, 1970-1985*

\begin{tabular}{|c|c|c|c|c|c|c|c|c|c|c|c|c|}
\hline & \multicolumn{4}{|c|}{1970} & \multicolumn{4}{|c|}{1976} & \multicolumn{4}{|c|}{1985} \\
\hline & \multicolumn{2}{|c|}{ PLQ } & \multicolumn{2}{|c|}{$\mathbf{P Q}$} & \multicolumn{2}{|c|}{ PLQ } & \multicolumn{2}{|c|}{$\mathbf{P Q}$} & \multicolumn{2}{|c|}{ PLQ } & \multicolumn{2}{|c|}{$\mathbf{P Q}$} \\
\hline & $\mathbf{N}$ & $\%$ & $\mathbf{N}$ & $\%$ & $\mathbf{N}$ & $\%$ & $\mathbf{N}$ & $\%$ & $\mathbf{N}$ & $\%$ & $\mathbf{N}$ & $\%$ \\
\hline Politique & 5 & 6,9 & - & - & 5 & 19,2 & 6 & 8,4 & 2 & 2,0 & 3 & 13,0 \\
\hline Social & 19 & 26,4 & 2 & 28,6 & 7 & 26,9 & 14 & 19,7 & 21 & 21,2 & 4 & 17,4 \\
\hline Culturel & 7 & 9,7 & 2 & 28,6 & 2 & 7,7 & 33 & 46,5 & 18 & 18,2 & 9 & 39,1 \\
\hline Administratif & 25 & 34,7 & 2 & 28,6 & 2 & 7,7 & 10 & 14,1 & 30 & 30,3 & 4 & 17,4 \\
\hline Économique & 16 & 22,2 & 1 & 14,3 & 10 & 38,5 & 8 & 11,3 & 28 & 28,3 & 3 & 13,0 \\
\hline Total & 72 & 99,9 & 7 & 100,1 & 26 & 100,0 & 71 & 100,0 & 99 & 100,0 & 23 & 99,9 \\
\hline
\end{tabular}


remarquer Daniel Gaxie en parlant de la situation française, le pôle économique est d'autant mieux représenté que l'on va, selon les taxinomies en vigueur au sein du champ politique, de la «gauche» vers la «droite» ou le «centre». À l'inverse, ajoutait-il, le pourcentage des députés issus des milieux intellectuels diminue quand on va de la «gauche» vers la «droite» ou le «centre» ${ }^{34}$.

Brigitte Gaïti en arrive à la même conclusion, tout en tenant compte des particularités de la situation en France où s'est opéré un processus de fusion entre l'exécutif et la haute administration qualifié de «République administrative» par Birnbaum. «Les gouvernements de droite, écrit-elle, ont été peuplés d" "hommes d'action", détenteurs de pouvoir économique et surtout politique (dans le sens de haute administration). La gauche a promu avant tout des "hommes de réflexion", des intellectuels "purs" que la logique institutionnelle de la $\mathrm{V}^{\mathrm{e}}$ République, à l'origine d'un processus de fusion entre l'exécutif et la Haute Administration, avait éloignés du centre du système politique. ${ }^{35}$

L'arrivée des intellectuels au pouvoir au Québec constitue ainsi le pendant du retour des intellectuels avec la venue de la gauche française au pouvoir en 1981. Mais dans l'un et l'autre cas on s'est buté à un obstacle de taille: la crise économique profonde des années 1982-1984, qui devait paver la voie au retour des gaullistes et giscardiens en France et des libéraux au Québec. Les intellectuels producteurs et surtout consommateurs d'idéologie ${ }^{36}$ ont dû faire place aux entrepreneurs et administrateurs portés par un nouveau courant idéologique qui valorise le secteur privé, le champ économique et le désengagement de l'État. Pour gérer le quotidien politique, on fait ainsi appel à des administrateurs ou à des gestionnaires plutôt qu'à des intellectuels plus aptes à concevoir des projets de société. En ce sens, la montée de l'entrepreneur-administrateur, qui vient remplacer l'intellectuel, correspond à l'importance croissante accordée à ce groupe au sein d'une société qui valorise davantage la «solution libérale», où la gérance du politique doit se conformer de plus en plus au mode de gestion en vigueur dans l'entreprise privée. L'analyse du personnel politique nous permettrait ainsi de détecter, à l'exemple du séismographe ${ }^{37}$, les changements profonds au sein d'une société.

34 Daniel Gaxie, op. cit., 30-31.

35 Brigitte Gaïti, op. cit., 64.

36 Selon les termes de François Bourricaud dans Le bricolage idéologique. Essai sur les intellectuels et les passions démocratiques (Paris, Presses universitaires de France, 1980), 8.

37 Selon l'expression de Robert D. Putnam, op. cit., 43. 
Quant à la période antérieure au réalignement de 1970, elle est marquée par la forte présence du champ économique au sein de l'Union nationale, bien qu'un député sur trois ou sur quatre soit issu du champ social durant toutes ces années (voir le tableau 5). Cette domination du champ économique ne doit pas nous surprendre puisque la députation unioniste était recrutée largement parmi les propriétaires de PME, petits industriels ou commerçants habituellement bien implantés dans leur milieu, et chez les professionnels du droit et de la santé (qui représentent surtout le champ social).

Le Parti libéral, pour sa part, se caractérise par le déclin constant du champ économique - prédominant en 1944 et très faible en 1966 -, par une forte présence du champ social et par une remontée constante du champ administratif. À nouveau, aussi bien pour le Parti libéral que pour l'Union nationale, l'élection de 1966 marque une rupture par rapport aux années antérieures si bien qu'un nouveau type de député s'installe alors à l'Assemblée nationale. Le petit entrepreneur et l'avocat qui avaient si nettement dominé la Chambre depuis 1944 vont céder progressivement la place à des professionnels de la culture et à de plus «gros» entrepreneurs, si bien qu'à la fin des années 1960 , les professionnels de toutes catégories (où les avocats sont toujours présents) vont constituer le groupe le plus nombreux à l'Assemblée. Cette mutation du personnel politique s'affirmera davantage avec l'arrivée du Parti québécois sur la scène politique.

En somme, si l'on peut opposer l'entrepreneur et l'intellectuel après l'élection de 1970, chacun étant mieux représenté dans un parti politique, il faut plutôt opposer l'entrepreneur et le travailleur du champ social au cours de la période antérieure, chacun étant davantage présent au sein d'un parti. Ce qui nous autorise à conclure, contrairement aux prétentions des théories élitistes, à la non-homogénéité des représentants durant ce demi-siècle de vie politique.

\section{CIRCULATION DES ÉLITES}

Non-représentativité et non-homogénéité, ces deux concepts réfêrent essentiellement à des attributs socio-économiques qui caractérisent l'élite politique. Mais celle-ci peut se maintenir longtemps au pouvoir ou changer rapidement, se reproduire ou se renouveler. On a habituellement associé un faible renouvellement du leadership à la stabilité institutionnelle et à la quiétude politique, alors que le renouvellement élevé est associé à des périodes de crise. C'est ainsi que les élections de réalignement s'accompagnent d'un renouvellement profond de la députation en ce sens que le taux de roulement y est 
TABLEAU 5

Champs d'activités des députés québécois selon le parti, 1944-1966*

\begin{tabular}{|c|c|c|c|c|c|c|c|c|c|c|c|c|c|c|c|c|}
\hline & \multicolumn{4}{|c|}{1944} & \multicolumn{4}{|c|}{1956} & \multicolumn{4}{|c|}{1960} & \multicolumn{2}{|c|}{1966} & \multirow{2}{*}{\multicolumn{2}{|c|}{ PLQ }} \\
\hline & \multicolumn{2}{|c|}{$\mathbf{U N}$} & \multicolumn{2}{|c|}{ PLQ } & \multicolumn{2}{|c|}{ UN } & \multicolumn{2}{|c|}{ PLQ } & \multicolumn{2}{|c|}{ UN } & \multicolumn{2}{|c|}{ PLQ } & \multicolumn{2}{|c|}{ UN } & & \\
\hline & $\mathbf{N}$ & $\%$ & $\mathbf{N}$ & $\%$ & $\mathbf{N}$ & $\%$ & $\mathbf{N}$ & $\%$ & $\mathbf{N}$ & $\%$ & $\mathbf{N}$ & $\%$ & $\mathbf{N}$ & $\%$ & $\mathbf{N}$ & $\%$ \\
\hline Politique & - & - & 1 & 2,7 & - & - & - & - & - & - & 1 & 2,0 & - & - & 1 & 2,0 \\
\hline Social & 16 & 33,3 & 14 & 37,8 & 21 & 29,2 & 6 & 30,0 & 11 & 25,6 & 18 & 35,3 & 15 & 26,8 & 20 & 40,0 \\
\hline Culturel & - & - & 2 & 5,4 & 1 & 1,4 & 2 & 10,0 & - & - & 3 & 5,9 & 7 & 12,5 & 5 & 10,0 \\
\hline Administratif & 7 & 14,6 & 2 & 5,4 & 12 & 16,7 & 4 & 20,0 & 8 & 18,6 & 11 & 21,6 & 11 & 19,6 & 15 & 30,0 \\
\hline Économique & 25 & 52,1 & 18 & 48,6 & 38 & 52,8 & 8 & 40,0 & 24 & 55,8 & 18 & 35,3 & 23 & 41,1 & 9 & 18,0 \\
\hline Total & 48 & 100,0 & 37 & 99,9 & 72 & 100,1 & 20 & 100,0 & 43 & 100,0 & 51 & 100,1 & 56 & 100,0 & 50 & 100,0 \\
\hline
\end{tabular}

* Voir le tableau 4 pour la définition des champs. 
élevés $^{38}$. À la «loi de l'inertie» de Mosca marquée par la tendance à la perpétuation de l'élite et à un faible renouvellement, on peut opposer des périodes de crise ou, tout au moins, de changements qui se traduisent par un renouvellement plus élevé de la classe politique.

L'ouverture de la classe politique peut prendre différentes formes $^{39}$. On peut la mesurer par le taux de roulement des députés qui marque l'arrivée d'une nouvelle classe politique au pouvoir en remplacement des anciennes élites. Mais ce critère nous indique avant tout que le personnel politique s'est renouvelé, qu'il y a eu circulation rapide au sein de l'élite, sans nous renseigner sur la nature du changement. Circulation des élites n'est pas synonyme de l'arrivée d'une classe politique différente. C'est pourquoi il faut aussi retourner aux caractéristiques socio-économiques des nouveaux élus par rapport à celles de l'ancienne classe dirigeante.

Pour analyser la circulation des élites, il importe de se replacer dans une perspective de longue durée afin de pouvoir dégager des périodes de stabilité et de continuité suivies de périodes de renouvellement rapide. Si Maurizio Cotta a pu étudier la relation entre le processus de reproduction et de renouvellement de la classe politique italienne et la discontinuité du régime démocratique, une telle perspective n'est pas possible dans le cas québécois, où domine le même type de régime politique depuis la Confédération de 1867. Par contre, on peut déceler ici des élections ou des périodes de réalignements électoraux qui se traduisent en même temps par un renouvellement du personnel politique et des transformations du leadership $^{40}$. Autour de l'élection de 1897 comme de celles de 1936 et de 1970 , nous assistons à des réalignements de l'électorat québécois et à un fort roulement du personnel, qui se manifeste également par des changements dans les caractéristiques socio-économiques de ce personnel (voir le tableau 6).

Les réalignements de 1897 et de 1936 ont été suivis, après une phase d'ajustement, par de longues périodes de stabilité où le renouvellement des parlementaires est faible et où le vieillissement se fait de plus en plus sentir. Si l'on s'arrête à la période plus récente marquée par la montée du Parti québécois, la disparition de l'Union nationale et la présence fugace du Ralliement créditiste, on ne perçoit pas une telle stabilité. Au contraire, la situation évolue en dents de scie, avec alternance de taux élevé et faible taux de roulement, et ces

38 Voir Réjean Pelletier et Jean Crête, «Réalignements électoraux et transformations du personnel politique», Revue canadienne de science politique, 21,1 (mars 1988): 3-33.

39 Maurizio Cotta, op. cit., 160.

40 Réjean Pelletier et Jean Crête, op. cit. 
TABLEAU 6

Renouvellement des députés québécois depuis 1919

\begin{tabular}{cccc}
\hline Élections & Députés élus & Nouveaux députés & Taux de roulement* \\
\hline 1919 & 81 & 23 & 28,4 \\
1923 & 84 & 30 & 32,1 \\
1927 & 85 & 25 & 29,4 \\
1931 & 90 & 25 & 23,5 \\
1935 & 90 & 46 & 51,1 \\
1936 & 90 & 42 & 46,7 \\
1939 & 86 & 60 & 69,8 \\
1944 & 91 & 48 & 50,0 \\
1948 & 92 & 36 & 38,5 \\
1952 & 92 & 22 & 23,9 \\
1956 & 93 & 28 & 29,3 \\
1960 & 95 & 36 & 36,6 \\
1962 & 95 & 29 & 30,5 \\
1966 & 108 & 54 & 43,2 \\
1970 & 108 & 57 & 52,8 \\
1973 & 110 & 40 & 35,2 \\
1976 & 110 & 79 & 71,8 \\
1981 & 122 & 44 & 29,1 \\
1985 & 122 & 63 & 51,6 \\
1989 & 125 & 34 & 25,4 \\
\hline
\end{tabular}

* Calculé selon la formule suivante:

NDx - NSx

NTx -1

NDx : nombre de nouveaux députés à l'élection $X$

NSx : nombre de nouveaux sièges à l'élection $X$

NTx - 1: nombre de députés à l'élection précédente

changements rapides sont favorables à l'émergence d'un leadership jeune et fortement scolarisé, appelé à céder rapidement la place à d'autres parlementaires. Une telle constatation infirme la conclusion de Rossi-Landi à l'effet que le libre jeu de la concurrence, en démocratie, n'entraîne pas de bouleversements, donnant l'exemple de l'Europe occidentale où de $70 \%$ à $90 \%$ des députés qui se présentent sauvegardent leur mandat ${ }^{41}$. Depuis une vingtaine d'années au Québec, la situation est certainement plus fluide: le renouvellement du personnel est constant et souvent très prononcé. Faudrait-il conclure à la manière de Rossi-Landi qui, dégageant une constante de l'histoire politique française, affirmait que «les Assemblées trop renouvelées sont vouées à la médiocrité politique»? ${ }^{42}$ Encore une fois, la situation

41 Guy Rossi-Landi, Les hommes politiques (Paris, Presses universitaires de France, 1973), 15.

42 Ibid, 13. 
québécoise prouve le contraire. Le renouvellement du personnel a permis l'émergence d'un leadership présentant des caractéristiques différentes, le plus souvent porteur d'idées nouvelles et de politiques nouvelles. Certes, on ne peut rejeter la possibilité d'un effet déstabilisant d'une circulation trop rapide des élites, comme ce fut le cas en Italie avant l'arrivée du régime fasciste de Mussolini ${ }^{43}$. Ce fut peutêtre le cas au milieu de la présente décennie au Québec où plusieurs ont réclamé la "paix politique»: absence de débats constitutionnels sous le prétexte que la constitution n'intéresse pas la population et est réservée aux seuls experts; absence de débats de fond sur le rôle de l'État dans la société québécoise sous le prétexte que l'État a atteint sa limite de croissance, qu'il engendre d'énormes déficits et que la tendance est à la privatisation. C'est pourquoi on s'est alors contenté de la gestion du quotidien en suivant le modèle de gestion en vigueur dans le secteur privé. L'intellectuel producteur et consommateur d'idéologie, définisseur de situations et de projets de société, a été remplacé par l'entrepreneur-administrateur appelé à gérer les affaires de l'État comme on gère les affaires privées.

\section{CONCLUSION}

Si l'on s'attache d'abord au phénomène de la non-représentativité, on peut conclure, à l'instar de Daniel Gaxie, que la compétition politique engendre la sélection sociale. En effet, les caractéristiques des parlementaires québécois en termes d'éducation et de secteurs occupationnels ne sont nullement «représentatives» ou semblables à celles de l'ensemble de la population québécoise à la même époque. La situation au Québec se rapprocherait ainsi du modèle de l'agglutination: les détenteurs du pouvoir politique sont également détenteurs d'un niveau élevé de scolarité, se recrutent chez les professionnels ou appartiennent au milieu des affaires et à l'administration. Surtout, la formation universitaire constitue le nouveau critère d'entrée dans l'élite politique québécoise au moment où se diversifient de plus en plus les types de profession. S'il reste encore important en nombre, l'avocat n'est plus le grand-prêtre de la vie politique québécoise. On peut également ajouter que cette double caractéristique - nécessité d'une formation universitaire et diversification des professions reproduit les changements profonds survenus au sein de la société québécoise depuis les années 1960. L'analyse du personnel politique nous permettrait ainsi de détecter, à la manière du séismographe, de tels changements.

Cependant, en dépit de ces traits communs, l'élite politique québécoise n'est pas complètement homogène. Depuis le réalignement de 1970, ce qui distingue le plus nettement le Parti québécois du Parti 
libéral, c'est la possession différenciée d'un capital culturel et d'un capital économique; ce qui distinguait le Parti libéral de l'Union nationale durant les années antérieures, c'était l'appartenance plus grande au champ social dans le premier cas et au champ économique dans le second.

Depuis surtout la crise économique de 1982-1984 et l'élection de 1985, l'intellectuel du Parti québécois, concepteur d'un projet de société et consommateur d'idéologie, a dû faire place à l'entrepreneur et à l'administrateur du Parti libéral, porté par un courant de pensée qui valorise le champ économique et la gestion du politique à la manière du secteur privé. On est ainsi passé de l'État-entrepreneur aux entrepreneurs-administrateurs à la tête de l'État.

On peut enfin ajouter que, depuis une vingtaine d'années, on assiste au Québec à un renouvellement rapide de l'élite politique, qui contraste fortement avec les longues périodes de stabilité qui ont caractérisé les régimes Taschereau et Duplessis. Contrairement aux réalignements de 1897 et de 1936, celui de 1970 n'a pas été suivi jusqu'ici d'une période de stabilité. Au contraire, le roulement des parlementaires québécois est fort important depuis 1966 de telle sorte que les carrières politiques sont habituellement assez courtes. Ce renouvellement constant s'est traduit par l'émergence d'un leadership jeune et fortement scolarisé. Malgré tout, après la turbulence politique de ce dernier quart de siècle, le Québec aspire peut-être à la «paix politique» qui viendrait ainsi marquer une pause pour les années à venir. Mais il ne faut pas oublier que la politique comporte toujours une double face: si elle est intégration et recherche de consensus, elle est aussi lutte et affrontements. À des périodes de paix politique peuvent succéder des périodes de plus grande turbulence; à des périodes de conservatisme social peuvent succéder des périodes de renouvellement socio-politique. 